\title{
Thematic interpretation of the Northern Dvina River floodplain meadow ecosystem using remote sensing
}

\author{
Alexey Volkov*, and Tatyana Parinova \\ Northern (Arctic) Federal University named after M.V. Lomonosov. Higher School of Natural \\ Sciences and Technologies. Department of biology, ecology and biotechnology. Arkhangelsk, \\ Russian Federation
}

\begin{abstract}
In this work we represent the possibility of using satellite images of medium spatial resolution for thematic interpretation of meadow ecosystems located in the flood plain of the Northern Dvina river. We have used multidimensional scaling to study the differences in spectral characteristics. Classes such as arable land, water, mineral soils, forests and swamps are most well-classified. However, the meadow ecosystems overlap with reed beds according to spectral characteristics. Therefore, for more accurate identification of meadows, it is recommended to use different vegetative indices, for example NDVI and $\mathrm{Cl}$ Green. As a result of the research, a map of the distribution of meadow ecosystems was created using GIS.
\end{abstract}

\section{Introduction}

One of the traditional tasks for geobotany is the mapping of vegetation, including meadow, which is the object of our many years of research. Large-scale data on areas of meadow ecosystems in the Russian Federation refer mainly to the 1960-1980th years and at the present time do not adequately reflect the current situation [1]. Meadows in the floodplain are of high natural and economic value. Among other ecosystems, they are particularly dynamic and undergo significant changes over time. Their areas, the degree of moistening, the composition of the herbage are constantly changing. Therefore, it is important to have a reliable and affordable way of remote meadow registration and evaluation of their areas.

The aim of this study is to identify criteria that allow deciphering the meadow communities and create a map of the floodplain of the Northern Dvina River. According to this, the following tasks are set: to determine the spectral characteristics of meadows from satellite images, to compare them with other classes of objects, to identify differences, to establish the relationship of spectral characteristics to the values of vegetation indices, and to conduct thematic interpretation with the subsequent compilation of a map of the flood plain of the Northern Dvina River.

\footnotetext{
Corresponding author: a.g.volkov@narfu.ru
} 


\section{Materials and Methods}

The meadows in the floodplain of the Severnaya Dvina River representing grassy communities with more or less contiguous herbage and formed mainly by long-term mesophilous plants [2] were chosen as the research subject.

We have used several data sources for thematic interpretation of meadow ecosystems.

Firstly, during field expeditions in 2011-2017, in the floodplain of the Severnaya Dvina River, we have detected meadows and located their coordinates. Later they were added on the satellite image and used as reference sites.

Secondly, we used satellite images from the Sentinel-2 (07/24/2017) with a spatial resolution of 10, 20 and $60 \mathrm{~m} /$ pixel, depending on the channel. All spectral channels from MSI were included in the analysis.

Thirdly, to improve the accuracy of the classification, we used satellite-derived data, such as the vegetation index. First of all, this is NDVI (1), which reflects the relative amount of photosynthetically active biomass (2). And also Cl Green (2) - the index of photosynthetic activity of the vegetation cover, used in assessing the chlorophyll $\mathrm{a}$ and $\mathrm{b}$ content in plant leaves $[3,4]$.

$$
\begin{gathered}
N D V I=(N I R-R E D) /(N I R+R E D) \\
C l \text { Green }=\text { NIR } / \text { Green }-1
\end{gathered}
$$

Where NIR - near infrared channel; RED - red channel; GREEN - green channel.

Using ArcGIS, we created a vector polygonal layer with known natural objects: meadows, arable land, forests, reed beds, marshes, water and anthropogenic. For each class we created 1000 random points and extract values from all data sets. Then we exported the data to csv file and analysed in R. Multidimensional scaling with Bray-Curtis distance used for searching the differences between classes. Multidimensional scaling makes it possible to visualize multidimensional feature data in a two-dimensional space of coordinates based on the distance matrix. In addition, it is possible to find a correlation with the factors, determine their influence on the differentiation of objects and display them in the form of vectors on the graph.

The interpretation was also carried out in R using the "random forest" method [5] which is an ensemble learning method for classification that operates by constructing a multitude of decision trees at training time and outputting the class that is the mode.

\section{Results}

The above classification of the satellite image revealed significant differences in the spectral characteristics of various deciphered objects. As expected, classes of arable land, water, mineral soils (such as sandbanks in the delta of the river), forests and marshes are best separated. At the same time, it is quite difficult to distinguish between meadow ecosystems and the so-called reed (Figure 1A), which are heavily waterlogged areas with periodic seasonal underflooding, where reed Phragmites australis (Cav.) Trin. ex Steud. L. predominates.

Vegetative indices can be used for more accurate interpretation of meadow ecosystems and reed thickets. In our work we used NDVI and $\mathrm{Cl}$ Green. In $\mathrm{R}$ we calculated the correlation coefficients between these indices and multidimensional scaling axes, containing compressed information on the spectral characteristics of the objects. Meadow communities as a whole have higher values of the applied indices (Figure 1B), which 
corresponds to more phytomass and chlorophyll content in meadow grass plants than in coarse phytomass of reeds.

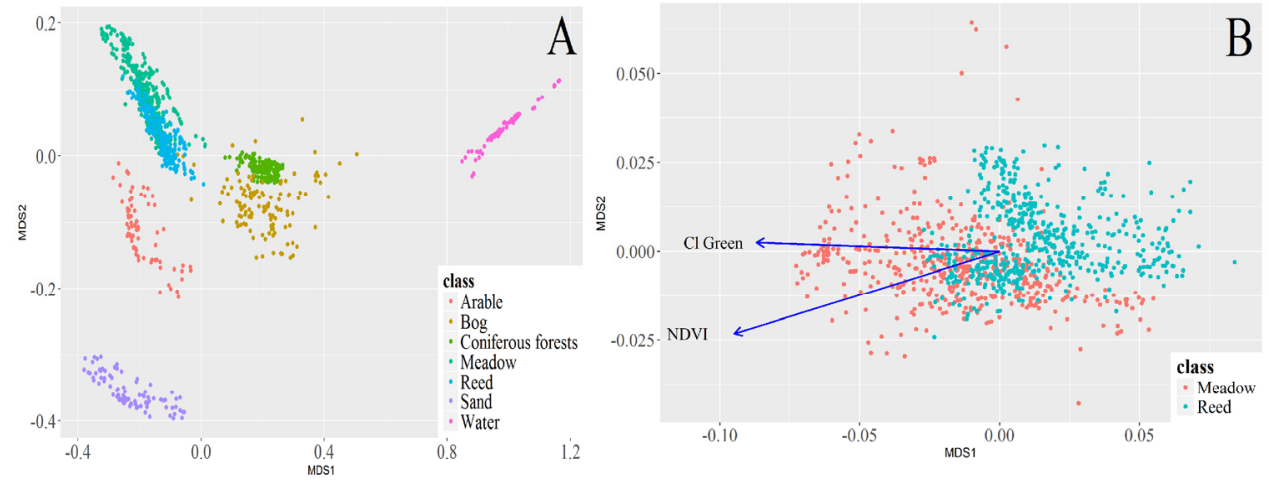

Fig. 1. The results of multidimensional scaling: A - for all classes of objects, B - for meadows and reed thickets with superposition of vectors of vegetative indices.

The obtained information on spectral characteristics and vegetation indices was used to decode the entire satellite image. Based on the results of the thematic interpretation, we have created a vector map of the meadow ecosystems of the floodplain of the Northern Dvina River.

The purpose of compiling any map is to present, at a certain scale, the objects of observation for their recording, as well as to study the patterns of their spread in connection with various natural factors. As a result of the study, spectral characteristics of meadow ecosystems located in the floodplain of the Northern Dvina River were determined. It is revealed that the meadows as a whole are well interpreted with respect to other classes of objects, such as forest, bog, arable and water. However, for the separation of meadows from classes with similar vegetation, it is necessary to use vegetative indices.

This project is supported by grant from the Russian Foundation for Basic Research (RFBR) and the government of Arkhangelsk region № 17-44-290111 (project manager: Prof. Elena Nakvasina).

\section{References}

1. T.K. Yurkovskaya, Actual problems of geobotany, 43-71 (Petrozavodsk, 2007)

2. B.M. Mirkin, L.M. Gareyeva, Bulletin of the Moscow Society of Naturalists 83, 38-50 (1978)

3. J. Rouse, R. Haas, J. Schell, D. Deering, 3rd ERTS Symposium, NASA SP-351, 1, 309-317 (1973)

4. G. Dall'Olmo, A.A. Gitelson, Applied Optics 44, 412-422 (2005)

5. T. Hastie, R. Tibshirani, J. Friedman, Random Forests. The Elements of Statistical Learning: Data Mining, Inference, and Prediction (Springer-Verlag, 2009) 